\title{
ORIGINAL ARTICLE \\ Establishing standard hospital performance measures for cervical spinal trauma: a Nationwide In-patient Sample study
}

\author{
DJ Hoh, M Rahman, KM Fargen, D Neal and BL Hoh
}

\begin{abstract}
Study design: A retrospective national administrative database study.
Objective: Patient safety indicators (PSIs) and hospital-acquired conditions (HACs) are metrics for quality of health care and are linked to reimbursement. The prevalence of PSIs/HACs may impact access to health care for certain conditions. We estimated the national occurrence rates of PSIs/HACs among cervical trauma patients and identified patient factors that correlate with their occurrence.
\end{abstract}

Setting: United States of America.

Methods: We queried Nationwide In-patient Sample database (NIS) hospitalizations (2002-2010) for diagnoses of cervical fracture with and without spinal cord injury (SCI). The incidence of each PSI/HAC was determined by ICD-9 (International Classification of Disease, 9th Revision) codes. Multivariate analysis was used to identify the correlation between specific variables and the probability of each indicator.

Results: There were 52377 hospitalizations for cervical fracture in the NIS (without SCI, $n=41$ 708; with SCl, $n=10669$ ). Among those without SCl, there were $5374(12.9 \%)$ reported PSIs and $117(0.3 \%)$ HACs. Leading adverse events were postoperative respiratory failure $(8.45 \%)$, pulmonary embolism $(1.70 \%)$ and pressure ulcer $(1.12 \%)$. Among those with $\mathrm{SCl}$, there were 6600 (61.9\%) PSIs and $143(1.3 \%) \mathrm{HACs}$. Leading adverse events were postoperative respiratory failure (39.2\%), pressure ulcer $(7.78 \%)$, sepsis (5.71\%), deep venous thrombosis (3.81\%) and PE (1.70\%). Adverse events were associated with several factors, including age, gender, Comorbidity Score and Injury Severity Score. Those with $\geqslant 1 \mathrm{PSI} / \mathrm{HAC}$ had significantly longer lengths of stay $(P<0.0001)$ and higher hospital costs $(P<0.0001)$ and mortality $(P<0.0001)$ compared with patients without events.

Conclusions: These results estimate baseline national rates of PSIs/HACs in patients with cervical spine trauma. These data may be used to gauge individual institutional quality of care in comparison with national data.

Spinal Cord (2016) 54, 306-313; doi:10.1038/sc.2015.185; published online 20 October 2015

\section{INTRODUCTION}

United States health care has made it a priority to improve the overall quality and safety of patient care. The impetus for this shift is credited to the 1999 Institute of Medicine report titled 'To Err is Human', where the Institute of Medicine estimated that 44 000-98 000 deaths occur annually in US hospitals owing to medical errors. ${ }^{1}$ In a subsequent call-to-action report titled 'The Quality Chasm', the Institute of Medicine urged the medical community to reduce medical errors by half. To enact these changes, US health care has looked toward specific patient safety indicators (PSIs) as measures of quality. The pay-for-performance ('value-based') paradigm is a reimbursement model, which then rewards institutions that meet benchmarks for these quality metrics. The Hospital Value-Based Purchasing Program, ${ }^{2}$ initiated by the Centers for Medicare and Medicaid Services (CMS), withholds $1 \%$ of reimbursement from a hospital's diagnosis-related group payments to then financially incentivize hospitals who provide better quality care.

These changes have coincided with increased public awareness of these indicators as a measure of individual hospital performance through publicly available databases, such as the US Department of
Health and Human Services Hospital Compare website. ${ }^{3}$ On these websites, individual hospitals can be queried and their rates of PSIs compared with national averages. Commonly referenced indicators include the Agency for Healthcare Research and Quality (AHRQ) PSIs, a series of metrics designed to report potential safety-related adverse events. ${ }^{4}$ Additionally, the CMS pay-for-performance program lists a number hospital-acquired conditions (HACs) that are designated as indicators of poor quality, and can prompt a denial for reimbursement. ${ }^{5}$ Taken together, these PSIs and HACs include a number of preventable patient care events, such as accidental puncture or patient falls, as well as potentially avoidable events such as central venous line (CVL) infection, deep venous thrombosis (DVT) or pulmonary embolism (PE).

As the US health-care system continues its transition toward valuebased reimbursement, the AHRQ PSIs and CMS HACs are becoming an important component of assessing the quality of care that hospitals provide, and will likely affect how hospitals are reimbursed. One concern, however, is that a consequence of this value-based reimbursement paradigm is the potential for restricted access to health care for certain medical conditions that pose higher risk for PSI- and HAC- 
related complications. Patients with traumatic cervical spinal cord injury (SCI) are particularly susceptible as they not only require significant health-care resources but are highly vulnerable to patient safety events such as PSIs and HACs. Unfortunately, there are currently few national data describing the rate of PSI/HACs for individual diagnosis codes by which institutions may compare their own performance. Also, there is a paucity of literature demonstrating which patient and hospital characteristics may affect PSI/HAC occurrence, which may be relevant given the varying hospital types and patient populations across the United States. To address this question, our specific aim was to estimate the national occurrence rate of PSIs and HACs among patients with cervical spine trauma with and without SCI using the Nationwide In-patient Sample database (NIS). Further, we sought to identify patient and hospital factors that correlate with these quality metrics, and determine the potential impact their occurrence may have on mortality, length of stay and cost outcomes.

\section{MATERIALS AND METHODS}

We obtained the NIS database from the AHRQ's Healthcare Cost and Utilization Project (Rockville, MD, USA). The NIS is a stratified systematic sample of hospitalization and discharges from all hospitals participating in the Healthcare Cost and Utilization Project (approximately equivalent to a random sample of $20 \%$ of all US nonfederal admissions, excluding rehabilitation and long-term acute care hospitals). NIS stratifies hospitals by region, location, teaching status, size and ownership. We followed methods outlined in the NIS documentation to account for this stratification in our estimates of the total number of hospitalizations for the years 2002-2010, and the national incidence of each indicator during that period. For more information regarding the NIS, please see http://www.hcup-us.ahrq.gov/nisoverview.jsp.

We searched the NIS for all teaching and nonteaching hospital admissions for cervical spinal trauma between 2002 and 2010. To specifically identify hospitalizations in the NIS for cervical spinal trauma, we used the ICD-9 (International Classification of Disease, 9th Revision) diagnosis codes for cervical fracture with SCI $(806.0,806.00-806.09,806.1,806.10-806.19)$ and cervical fracture without SCI $(805.0,805.00-805.08,805.10-805.18)$. Separate analyses were performed on the basis of the presence or absence of SCI. The incidence of a particular PSI or HAC among these hospitalizations was determined by summing the number of hospital records that included the ICD-9 code(s) indicating the presence of each PSI/HAC for each group. The quality indicator for 'Falls and trauma' was excluded from analysis as it was not possible to differentiate between a diagnosis code upon admission versus one occurring during hospitalization (i.e. the diagnosis code for 'Falls and trauma' occurred in $100 \%$ of patients queried for cervical fracture either with or without spinal cord injury, thereby not distinguishing between this specific quality indicator as being present on admission or not).

\section{Statistical methods}

We used he SAS statistical software package (version 9.3, SAS Institute Inc., Cary, NC, USA) to calculate means, standard deviations and frequencies for all patient and hospital characteristics and to estimate all PSI/HAC incidences. For incidences $<1 \%$, we constructed confidence intervals using the Agresti-Coull method. ${ }^{6}$

We performed multivariate analysis of all indicators that had an observed incidence rate $>0.1 \%$. We generated an overall Comorbidity Score for use as a covariate by summing the number of Elixhauser comorbidities ${ }^{7}$ recorded in the NIS for each hospitalization. We also generated an Injury Severity Score (ISS) as a covariate using methods developed by Clark et al. ${ }^{8}$ We used generalized estimating equations (SAS PROC GENMOD, version 9.3) to assess possible associations between patient and hospital characteristics and PSI/HACs. In all models, the presence of the indicator (yes or no) was taken as the outcome variable. The patient's age, gender, Comorbidity Score and ISS, as well as the hospital type (teaching versus nonteaching), size (small, medium, large) and region (northeast, midwest, south, west), were included as covariates. Results for hospital region were largely uninformative, showing no clear patterns, and are omitted. For all models, we graphically assessed the relationship between the probability of having the indicator and age, Comorbidity Score, ISS and hospital type and size. ${ }^{9,10} \mathrm{~A}$ linear model assumes a linear relationship between a continuous or ordered independent variable and the response variable. To determine whether this assumption was reasonable, we plotted the observed probability of experiencing the indicator by the deciles of age, Comorbidity Score and ISS, and by the ordered categories of hospital size. For age, Comorbidity Score and ISS, if this graphical assessment suggested a nonlinear association (such as increasing probability of the event with Comorbidity Score, but a plateau effect after a certain number of comorbidities), we modeled that relationship by using a quadratic or other nonlinear term. If a linear assumption was not plausible for hospital bed size, we modeled this variable as categorical. We used these results and the quasi-likelihood information fit criterion for generalized estimating equation models to determine whether any particular covariate modification (typically, adding a quadratic term in age, or treating hospital size as an ordered rather than a nominal variable) resulted in a better fit than the baseline model. We assumed a binary distribution for the outcome variable, with a logit link function. To account for the clustering of observations on hospitals, we treated hospital as a repeated factor, and we assumed an exchangeable working correlation. Finally, mean length of stay, hospital charges (adjusted 3\% per year for inflation) and mortality were compared between those with one or more PSI/HAC during their hospital stay and those with no PSI or HAC. Statistical significance was determined by a $P$-value $<0.05$.

\section{RESULTS}

The NIS query resulted in 52377 admissions for cervical spinal fracture (without SCI, $n=41708$; with SCI, $n=10669$ ). Patient and hospital characteristics are listed in Table 1. The incidence of PSI/HACs is listed in Table 2.

Among these hospitalizations for patients without SCI, there were $5374(12.9 \%)$ PSIs and $117(0.3 \%)$ HACs, with $10.9 \%$ of patients experiencing one or more PSI/HACs. Leading adverse events were postoperative respiratory failure $(8.45 \%)$, pulmonary embolism $(\mathrm{PE})$ $(1.70 \%)$ and pressure ulcer $(1.12 \%)$. Among these hospitalizations for patients with SCI, there were 6600 (61.9\%) PSIs and $143(1.3 \%)$

Table 1 Patient and hospital characteristics for patients with cervical fracture $^{a}$

\begin{tabular}{lccc}
\hline Characteristic & $\begin{array}{c}\text { Without SCI } \\
(\mathrm{n}=41 \text { 708) }\end{array}$ & $\begin{array}{c}\text { With SCI } \\
(\mathrm{n}=10669)\end{array}$ & $\mathrm{P}$ \\
\hline Age (years) (mean \pm s.d.) & $56.7 \pm 24.4$ & $47.7 \pm 22.3$ & $<0.0001$ \\
& & & \\
Gender & & & \\
$\quad$ Female & $17937(43.2 \%)$ & $2900(27.2 \%)$ & $<0.0001$ \\
$\quad$ Male & $23626(56.8 \%)$ & $7761(72.8 \%)$ & \\
& & & \\
$\begin{array}{l}\text { Comorbidity Score } \\
\text { (mean } \pm \text { s.d., median) }\end{array}$ & $1.5 \pm 1.6,1.0$ & $1.5 \pm 1.5,1.0$ & 0.158 \\
$\begin{array}{l}\text { ISS (mean } \pm \text { s.d., median) } \\
\text { Hospital type }\end{array}$ & $7.0 \pm 5.4,5.0$ & $22.5 \pm 16.9,17.0$ & $<0.0001$ \\
$\quad$ Nonteaching & $12643(30.6 \%)$ & $2529(23.9 \%)$ & $<0.0001$ \\
$\quad$ Teaching & $28675(69.4 \%)$ & $8046(76.1 \%)$ & \\
& & & \\
Hospital bedsize & & & \\
$\quad$ Small & $2071(5.0 \%)$ & $443(4.2 \%)$ & $<0.0001$ \\
$\quad \begin{array}{l}\text { Medium } \\
\text { Large }\end{array}$ & $8600(20.8 \%)$ & $2000(18.9 \%)$ & \\
\hline
\end{tabular}

Abbreviations: ISS, Injury Severity Score; SCI, spinal cord injury.

aWithout $\mathrm{SCl}(n=41708)$ and with $(n=10669) \mathrm{SCl}$. 
Table 2 Incidence (with $95 \% \mathrm{CI}$ ) of PSIs and HACs in patients with cervical fracture ${ }^{\mathrm{a}}$

\begin{tabular}{|c|c|c|c|c|}
\hline \multirow[t]{2}{*}{ Indicator } & \multicolumn{2}{|c|}{ Without SCI $(\mathrm{n}=41$ 708) } & \multicolumn{2}{|c|}{ With SCl $(\mathrm{n}=10669)$} \\
\hline & $\begin{array}{l}\text { Number with } \\
\text { condition }\end{array}$ & $\begin{array}{c}\text { Estimated national } \\
\text { rate }(95 \% \mathrm{Cl})\end{array}$ & $\begin{array}{l}\text { Number with } \\
\text { condition }\end{array}$ & $\begin{array}{c}\text { Estimated national } \\
\text { rate }(95 \% \mathrm{Cl})\end{array}$ \\
\hline \multicolumn{5}{|l|}{ Patient safety indicators } \\
\hline Anesthetic complications-therapeutic & b & b & b & b \\
\hline Anesthetic complications-poisoning & b & $\mathrm{b}$ & $\mathrm{b}$ & b \\
\hline latrogenic pneumothorax & 56 & $0.13 \%(0.103 \%, 0.175 \%)$ & 69 & $\begin{array}{l}0.65 \%(0.510 \% \\
0.819 \%)\end{array}$ \\
\hline Central venous line infection & 67 & $0.16 \%(0.126 \%, 0.204 \%)$ & 108 & $1.01 \%(0.838 \%, 1.22 \%)$ \\
\hline Postoperative hip fracture & 231 & $0.554 \%(0.487 \%, 0.630 \%)$ & 118 & $1.11 \%(0.924 \%, 1.32 \%)$ \\
\hline Postop physiologic derangement-secondary diabetes with ketoacidosis & b & $\mathrm{b}$ & b & b \\
\hline Postop physiologic derangement—diabetes with ketoacidosis & 11 & $\begin{array}{l}0.03 \%(0.0141 \% \\
0.0479 \%)\end{array}$ & b & b \\
\hline Postoperative respiratory failure & 3525 & $8.45 \%(8.19 \%, 8.72 \%)$ & 4181 & $39.20 \%(38.3 \%, 40.1 \%)$ \\
\hline Deep vein thrombosis & 372 & $0.89 \%(0.806 \%, 0.987 \%)$ & 407 & $3.81 \%(3.45 \%, 4.20 \%)$ \\
\hline Pulmonary embolism & 142 & $1.70 \%(1.47 \%, 1.96 \%)$ & 181 & $1.70 \%(1.47 \%, 1.96 \%)$ \\
\hline Sepsis & 380 & $0.34 \%(0.289 \%, 0.401 \%)$ & 609 & $5.71 \%(5.28 \%, 6.16 \%)$ \\
\hline Postop wound dehiscence & $\mathrm{b}$ & $\mathrm{b}$ & $\mathrm{b}$ & $\mathrm{b}$ \\
\hline Accidental puncture or laceration & 62 & $0.15 \%(0.116 \%, 0.191 \%)$ & 50 & $\begin{array}{l}0.47 \%(0.354 \% \\
0.618 \%)\end{array}$ \\
\hline Transfusion reaction & $\mathrm{b}$ & $\mathrm{b}$ & $\mathrm{b}$ & b \\
\hline \multicolumn{5}{|l|}{ Hospital-acquired conditions } \\
\hline Manifestations of poor glycemic control—diabetic ketoacidosis & 11 & $\begin{array}{l}0.03 \%(0.0141 \% \\
0.0479 \%)\end{array}$ & $\mathrm{b}$ & b \\
\hline Manifestations of poor glycemic control—nonketotic hyperosmolar coma & b & b & b & b \\
\hline Manifestations of poor glycemic control—hypoglycemic coma & b & b & b & b \\
\hline Manifestations of poor glycemic control—secondary diabetes with ketoacidosis & b & b & b & b \\
\hline Manifestations of poor glycemic control—secondary diabetes with hyperosmolarity & b & b & b & b \\
\hline Surgical site infection, mediastinitis, following coronary artery bypass graft & b & b & b & b \\
\hline Surgical site infection following certain orthopedic procedures & 45 & $0.11 \%(0.0803 \%, 0.145 \%)$ & 62 & $\begin{array}{l}0.58 \%(0.453 \% \\
0.745 \%)\end{array}$ \\
\hline Surgical site infection following bariatric surgery for obesity & b & b & b & b \\
\hline $\begin{array}{l}\text { Deep vein thrombosis and pulmonary embolism following certain orthopedic } \\
\text { procedures }\end{array}$ & b & b & b & b \\
\hline
\end{tabular}

Abbreviations: $\mathrm{Cl}$, confidence interval; $\mathrm{HAC}$, hospital-acquired condition; $\mathrm{PSI}$, patient safety indicator; SCl, spinal cord injury.

Abbreviations: $\mathrm{Cl}$, confidence interval; $\mathrm{HAC}$, hospital-acqu
aWithout SCI $(n=41708)$ and with $\mathrm{SCl}(n=10669)$.

${ }^{\mathrm{b}} \mathrm{A}$ small number of patients $(n \leqslant 10)$.

HACs. Leading adverse events were postoperative respiratory failure (39.2\%), pressure ulcer $(7.78 \%)$, sepsis $(5.71 \%)$, DVT $(3.81 \%)$ and PE (1.70\%), with $46.3 \%$ of patients experiencing one or more PSI/HACs.
Effect of patient characteristics on PSI/HAC incidence

For patients without SCI, the effect of age, gender, Comorbidity Score and ISS on the incidence of PSI/ HACs is displayed in Table 3. Increasing age was linearly correlated with higher risk for 


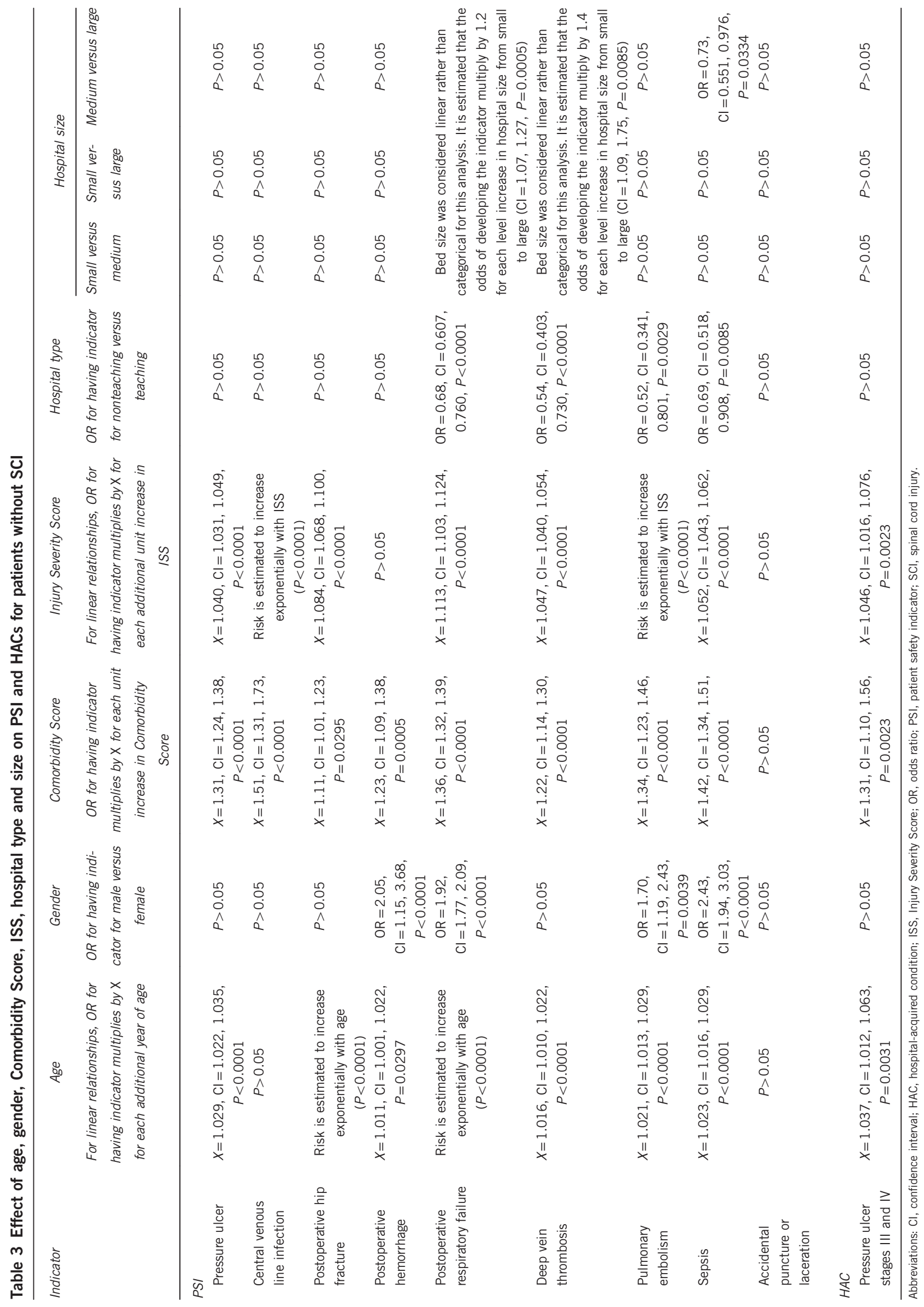


pressure ulcer, postoperative hemorrhage, DVT, PE and sepsis. Risk increased exponentially with increasing age for postoperative hip fracture and postoperative respiratory failure. Male gender was linearly correlated with increased risk for postoperative hemorrhage, postoperative respiratory failure, PE and sepsis. Higher Comorbidity Score was associated with increased risk for pressure ulcer, CVL infection, postoperative hip fracture, postoperative hemorrhage, postoperative respiratory failure, DVT, PE and sepsis. Higher ISS was linearly correlated with increased risk for pressure ulcer, postoperative hip fracture, postoperative respiratory failure, DVT and sepsis. Risk for CVL infection and PE increased exponentially with increasing ISS.

For patients with SCI, the effect of individual patient characteristics on the incidence of PSI/HACs is displayed in Table 4. Increasing age was linearly correlated with higher risk for pressure ulcer and postoperative hip fracture. Risk for postoperative hemorrhage and DVT increased exponentially until age 50 years, and then decreased exponentially with older age. Risk for PE and surgical site infection exponentially increased until age 41 and 45 years, respectively, and then decreased thereafter. Male gender was linearly correlated with increased risk for pressure ulcer, postoperative respiratory failure, $\mathrm{PE}$ and sepsis. Higher Comorbidity Score was associated with increased risk for pressure ulcer, CVL infection, postoperative hip fracture, postoperative hemorrhage, postoperative respiratory failure, DVT, PE and sepsis. Higher ISS was linearly correlated with increased risk for pressure ulcer, CVL infection, postoperative respiratory failure, DVT, $\mathrm{PE}$ and sepsis. Risk for postoperative hip fracture increased exponentially with increasing ISS.

\section{Effect of hospital type and size on PSI/HAC incidence}

For patients without SCI, the effect of hospital type and size on the incidence of PSI/HACs in multivariate analysis is displayed in Table 3. Nonteaching hospitals were associated with a lower risk for postoperative respiratory failure, DVT, PE and sepsis. Hospital size was linearly correlated with increasing risk for postoperative respiratory failure and DVT, for each level size increase from small to large. Medium hospital size was associated with a lower risk for sepsis compared with large hospitals.

For patients with SCI, the effect of hospital characteristics on the incidence of PSI/HACs in multivariate analysis is displayed in Table 4. Nonteaching hospitals were associated with a lower risk for pressure ulcer, postoperative hemorrhage, postoperative respiratory failure, $\mathrm{PE}$, sepsis and accidental puncture or laceration. Hospital size was linearly correlated with increasing risk for DVT and sepsis, for each level size increase from small to large. Risk for postoperative respiratory failure was lower for small versus medium or large hospital size. Risk for PE was lower for medium versus large hospital size. Risk for pressure ulcer stages III and IV was higher for small versus large hospital size.

Effect of PSI/HAC on length of stay, mortality and hospital charges The effect of PSI/HACs on outcome measures is displayed in Table 5. Patients without SCI, and who had $\geqslant 1$ PSI/HAC, had significantly longer mean lengths of stay (16.4 versus 4.5 days, $P<0.0001)$, higher mortality rates $(17.2 \%$ versus $1.3 \%, P<0.0001)$ and higher mean hospital charges (US $\$ 158800$ versus US $\$ 41000, P<0.0001$ ) compared with patients without a PSI/HAC. Patients with SCI, and who had $\geqslant 1$ PSI/HAC, had significantly longer mean lengths of stay (26.6 versus 10.7 days, $P<0.0001)$, higher mortality rates $(25.7 \%$ versus $4.1 \%, P<0.0001$ ) and higher mean hospital charges (US
$\$ 262500$ versus US\$103600, $P<0.0001)$ compared with patients without a PSI/HAC.

\section{DISCUSSION}

The purpose of this study was to estimate the national occurrence rate of PSIs and HACs among patients hospitalized for cervical spinal fracture either with or without SCI, and to identify patient and hospital factors that correlated with their occurrence. The data reveal that, for these two specific populations, the occurrence of PSIs/HACs is relatively high, indicating the complexity of the underlying conditions and the vulnerability of patients to suffer from one or more of these events. Among 41708 admissions for cervical fracture without SCI, there were 5374 PSIs with an incidence of $12.9 \%$. More notably, in 10669 admissions for cervical fracture with SCI, there were 6600 PSIs. The incidence of PSIs in this susceptible population was $61.9 \%$, with nearly $40 \%$ having respiratory failure. The incidence of PSIs in both groups was significantly affected by multiple patient and hospital factors, including age, comorbidities, injury severity, hospital type and size. Age, Comorbidity and ISSs appear to have the largest effect on PSIs in this patient population. This would suggest that hospitals caring for disproportionately high numbers of elderly, systemically ill, complex and/or spinal cord-injured patients are likely to have higher PSIs compared with those caring for younger, healthier and neurologically normal patients.

We additionally sought to determine the potential impact of having one or more PSIs/HACs on mortality, length of stay and cost outcomes. The occurrence of a single PSI or HAC had a significant association with each of these measures. A single PSI/HAC event was associated with more than 11 additional hospital days, a 4 (without SCI)- and 2.5 (with SCI)-fold increase in hospital charges, and a 13 (without SCI)- and 6 (with SCI)-fold increase in mortality compared with those without a PSI/HAC during hospitalization. Although this statistical analysis cannot determine a causal link between harm events and worse outcome measures, this analysis does indicate that further evaluation of this association will be an integral step in realizing the importance of PSI/HACs in future quality and reimbursement initiatives. The potential impact of PSI/HACs on reimbursement and consequently access to health care is most relevant for those with SCI. Current comprehensive acute and long-term medical care for the SCI population has demonstrated major improvements in reducing morbidity and increasing overall life expectancy; however, with a significant burden on health-care resources. Therefore, one must consider the effect of a 'value-based' reimbursement paradigm on possibly restricting resource-limited SCI medical care for affected individuals.

The AHRQ PSIs were created as part of a Department of HHS initiative to improve patient safety through three mechanisms: by serving as a screening instrument to detect potential patient safety problems, offering insight into the adverse impact of medical errors and providing benchmarks for tracking progress in patient safety effort. Since the publication of the AHRQ PSIs, the validity of PSIs as measures of quality of care has been evaluated in a number of settings. ${ }^{11-16}$ Currently, the role of PSIs is greatest in publicly available hospital comparison websites, such as Hospital Compare, ${ }^{2}$ but PSIs have yet to play a role in CMS reimbursement. Although the AHRQ PSIs are not included in the new CMS Hospital Value-Based Purchasing Program, health-care professionals continue to debate the validity of generalizing such broad measures to hospitals with very different patient populations. Further, the ability of PSIs to accurately differentiate pre-existing from HACs has come into question. ${ }^{16}$ Because of the implications of PSI and HAC rates for hospitals and 


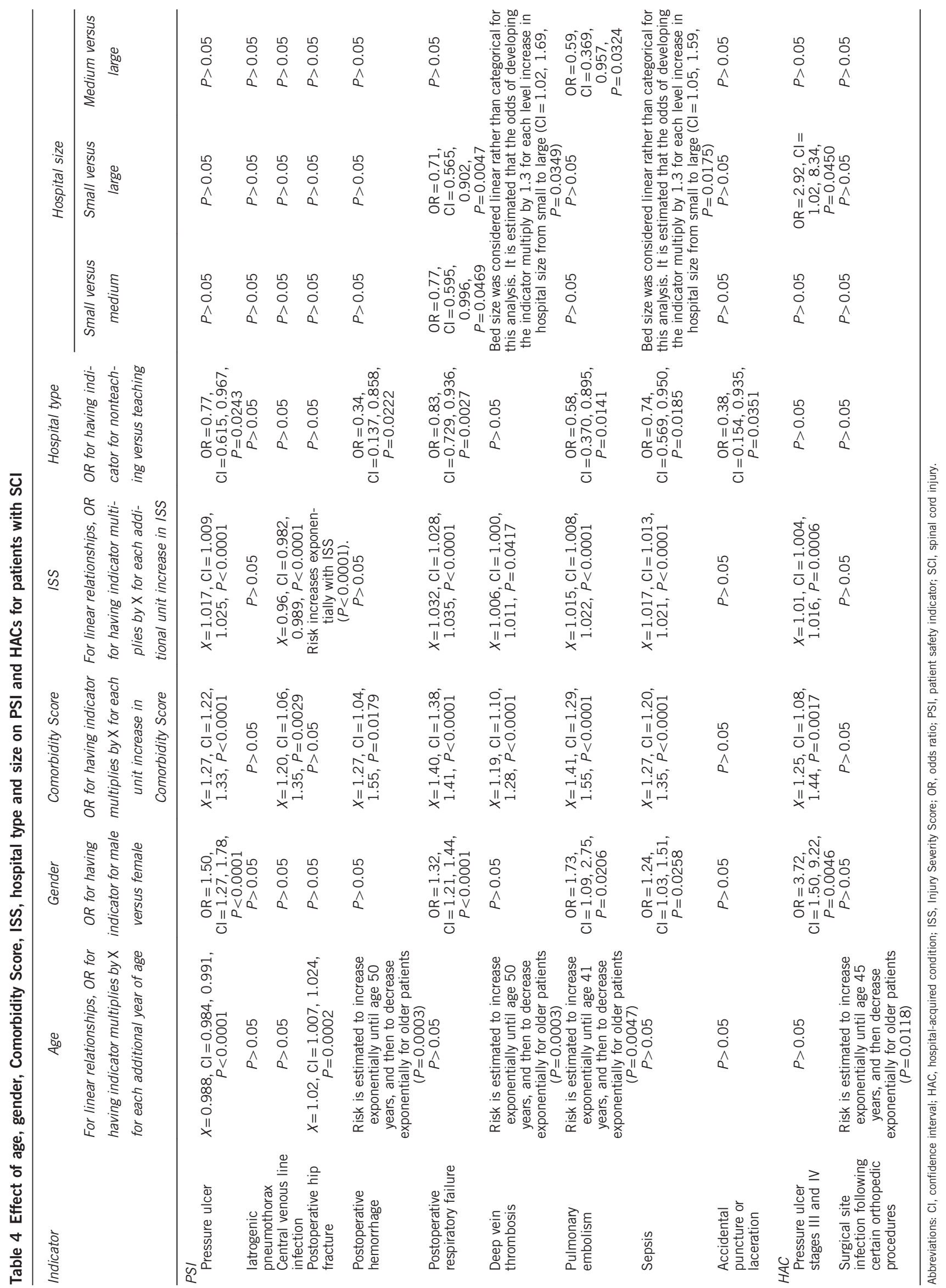


Table 5 Effect of PSIs and HACs on length of stay, mortality and hospital charges ${ }^{\mathrm{a}}$

\begin{tabular}{lccc}
\hline & No PSI or HAC & $\geqslant 1$ PSI or HAC & P \\
\hline $\begin{array}{l}\text { Without SCI } \\
\text { Length of stay in } \\
\text { days (mean } \pm \text { s.d.) }\end{array}$ & $4.5(5.0)$ & $16.4(17.0)$ & $<0.0001$ \\
Mortality & $\begin{array}{c}\text { 472/37 095 died } \\
(1.3 \%)\end{array}$ & $\begin{array}{c}779 / 4521 \text { died } \\
(17.2 \%)\end{array}$ & $<0.0001$ \\
& $41000(50000)$ & $158800(166200)$ & $<0.0001$ \\
$\begin{array}{l}\text { Hospital charges } \\
\text { (US\$) (mean } \pm \text { s.d.) }\end{array}$ & & & \\
$\begin{array}{l}\text { With SCI } \\
\text { Length of stay in } \\
\text { days } \\
\text { (mean } \pm \text { s.d.) }\end{array}$ & $10.7(14.2)$ & $26.6(29.8)$ & $<0.0001$ \\
Mortality & $236 / 5694$ & $1262 / 4911$ & $<0.0001$ \\
$\begin{array}{l}\text { Hospital charges } \\
\text { (US } \$ \text { ) (mean } \pm \text { s.d.) }\end{array}$ & $103600(119000)$ & $262500(235000)$ & $<0.0001$ \\
\hline
\end{tabular}

Abbreviations: $\mathrm{HAC}$, hospital-acquired condition; PSI, patient safety indicator; $\mathrm{SCl}$, spinal cord injury.

aExcluding hospitalizations with "Falls and trauma" as the only indicator.

physicians in terms of reimbursement and ratings, significant effort is now being invested in accurately coding patient preadmission risk profiles and in developing system-based practices that may prevent such in-patient harm events. Standardized procedural protocols have been highly successful in reducing the number of patient harm events. ${ }^{17-19}$

As the US health-care system continues its paradigm shift toward value-based reimbursement, PSIs may become an important addition to other quality indicators, such as those currently used by CMS, for determining reimbursement. Therefore, examination of the incidence of such PSIs within potentially susceptible patient populations, such as those with cervical trauma, is important for determining nationwide rates and associated risk factors. Currently, the University HealthSystem Consortium, an alliance of 120 academic medical centers and their affiliates that collect and report quality data for comparison, and publicly available online data such as the Department of Health and Human Services Hospital Compare website represent avenues for hospital-specific comparisons to national data. The NIS appears to represent an additional data set that may become increasingly helpful in cataloging national harm event trends, and for establishing guidelines for quality goals among institutions. Further, the NIS appears to be helpful in assessing pertinent associations between health-care cost, outcome measures and quality of care.

Limitations of this study include its retrospective nature and the inherent potential for selection bias. The NIS also does not provide clinical data with regard to certain patient factors and outcomes that may have offered even greater insight, such as patient neurologic and functional outcome, or intensive care unit length of stay. The NIS is also subject to coding errors and variability in coding. Our data are limited as the NIS does not allow distinction between preadmission conditions and hospital-acquired diagnoses. Therefore, some of these reported PSI/HACs may have been present on admission and mistakenly identified as new events.

Further, we did not attempt an analysis comparing the incidence of quality indicators in those with and without SCI as these represent different patient populations with multiple confounding factors besides the presence of neurologic deficit. One can speculate, however, on the basis of these data that the presence of a neurologic injury does impact the likelihood of developing an adverse event. Many of these are likely related to the underlying neurologic deficit (such as respiratory failure), whereas others may suggest the implicit challenges in treating patients with significantly impaired function (e.g. pressure ulcers, DVT, PE).

\section{CONCLUSION}

Health care in the United States is looking to various patient safety and quality metrics as measures of hospital performance, and linking reimbursement and penalties to their occurrence in order to prioritize higher standards of care. Cervical spinal trauma with and without spinal cord injury is shown to have a relatively common occurrence of PSIs and HACs, perhaps underscoring the complexity of these underlying conditions. The occurrence of a single PSI or HAC is also associated with a significant increase in length of stay, mortality and hospital charges. These data may serve as benchmarks by which individual hospitals may compare their performance against national standards. Also, given the relatively high rate of these PSIs and HACs in this susceptible population, hospitals that provide care for a disproportionately higher number of cervical spinal trauma patients may be more greatly impacted by these changes to the US health-care system.

\section{DATA ARCHIVING}

There were no data to deposit.

\section{CONFLICT OF INTEREST}

The authors declare no conflict of interest.

\section{ACKNOWLEDGEMENTS}

This work was not funded by the NIH, Wellcome Trust, HHMI or any other funding source.

1 Stelfox HT, Palmisani S, Scurlock C, Orav EJ, Bates DW. The 'To Err is Human' report and the patient safety literature. Qual Saf Health Care 2006; 15: 174-178.

2 Hospital Value-Based Purchasing Program: Fact Sheet. Available at: http://www.cms. gov/Outreach-and-Education/Medicare-Learning-Network-MLN/MLNProducts/ downloads/Hospital_VBPurchasing_Fact_Sheet_ICN907664.pdf (accessed on 15 October 2014).

3 Hospital Compare. US Department of Health and Human Services. Available at: http://www. hospitalcompare.hhs.gov/ (accessed on 15 October 2014).

4 Agency for Healthcare Research and Quality quality indicators. Available at: http://www.qualityindicators.ahrq.gov/ (accessed on 15 October 2014).

5 Hospital-acquired conditions. Available at: http://www.cms.gov/Medicare/Medicare-Feefor-Service-Payment/HospitalAcqCond/Hospital-Acquired_Conditions.html (accessed on 15 October 2014).

6 Agresti A, Coull BA. Approximate is better than 'Exact' for interval estimation of binomial proportions. Am Stat 1998; 52: 119-126.

7 Elixhauser A, Steiner C, Harris DR, Coffey RM. Comorbidity measures for use with administrative data. Med Care 1998; 36: 8-27.

8 Clark DE, Osler TM, Hahn DR. ICDPIC: Stata module to provide methods for translating International Classification of Diseases (Ninth Revision) diagnosis codes into standard injury categories and/or scores, 2010. Available at: http://ideas.repec.org/c/boc/bocode/ s457028.html (accessed on 15 October 2014).

9 Harrell FJ. Regression Modeling Strategies with Applications to Linear Models, Logistic Regression and Survival Analysis. Springer: New York, NY, USA, 2001.

10 Pan W. Akaike's information criterion in generalized estimating equations. Biometrics 2001; 57: 120-125.

11 Rhee D, Zhang Y, Papandria D, Ortega G, Abdullah F. Agency for Healthcare Research and Quality Pediatric Indicators as a quality metric for surgery in children: do they predict adverse outcomes? J Pediatr Surg 2012; 47: 107-111.

12 Sedman A, Harris JM II, Schulz K, Schwalenstocker E, Remus D, Scanlon M et al. Relevance of the Agency for Healthcare Research and Quality Patient Safety Indicators for children's hospitals. Pediatrics 2005; 115: 135-145.

13 Chang DC, Handly N, Abdullah F, Efron DT, Haut ER, Haider AH et al. The occurrence of potential patient safety events among trauma patients: are they random? Ann Surg 2008; 247: 327-334. 
14 Rosen AK, Rivard P, Zhao S, Loveland S, Tsilimingras D, Christiansen CL et al. Evaluating the patient safety indicators: how well do they perform on Veterans Health Administration data? Med Care 2005; 43: 873-884.

15 Vartak S, Ward MM, Vaughn TE. Do postoperative complications vary by hospital teaching status? Med Care 2008; 46: 25-32.

16 Bahl V, Thompson MA, Kau TY, Hu HM, Campbell DA Jr. Do the AHRQ patient safety indicators flag conditions that are present at the time of hospital admission? Med Care 2008; 46: 516-522.
17 Pronovost PJ, Marsteller JA, Goeschel CA. Preventing bloodstream infections: a measurable national success story in quality improvement. Health Aff (Millwood) 2011; 30: 628-634.

18 Centers for Disease Control and Prevention (CDC). Vital signs: central line-associated blood stream infections-United States, 2001, 2008, and 2009. Morb Mortal Wkly Rep 2011; 60: 243-248.

19 Rahman M, Whiting JH, Fauerbach LL, Archibald L, Friedman WA. Reducing ventriculostomy-related infections to near zero: the eliminating ventriculostomy infection study. Jt Comm J Qual Patient Saf 2012; 38: 459-464. 